\title{
Time-of-Flight Estimation using extended Matched Filtering
}

\author{
F. van der Heijden ${ }^{\dagger}$, S. van Koningsveld ${ }^{\ddagger}$, P.P.L. Regtien ${ }^{+}$ \\ ${ }^{\dagger}$ University of Twente, The Netherlands, F.vanderHeijden@ @ utwente.nl \\ ${ }^{\ddagger}$ Xsens Technologies bv, The Netherlands, s.vankoningsveld@ home.nl \\ ${ }^{+}$University of Twente, The Netherlands, p.p.l.regtien@utwente.nl
}

\begin{abstract}
The problem considered is the estimation of the ToF (timeof-flight) of an acoustic tone burst in a reflective environment. Secondary echoes cause a complex interference pattern. Only the ToF of the first echo is of interest. Conventional matched filtering (MF) cannot cope with overlapping echoes. An explicit model for overlapping echoes leads to a generalized MF consisting of a parallel bank of filters rather than just a single filter. The new method is evaluated with a dataset of 150 records of observed waveforms using 3-fold cross validation.
\end{abstract}

\section{Keywords}

ToF estimation, reflection model, time-of-flight

\section{INTRODUCTION}

Acoustic position and distance measurement systems are based on the principle of the Time-of-Flight (ToF) of a waveform. These systems consist of transmitter/receiver combinations which are geometrically arranged such that the distance between the object of interest and some reference position is proportional to the ToF, i.e. proportional to the time between transmitting and receiving the waveform.

The waveform that is used can have various forms, e.g. continuous wave, chirped FM, phase keyed. This paper is restricted to systems using tone bursts, i.e. a number of periods of a sine wave. The bandwidth of a tone burst can be kept moderate. Therefore, this signal is suitable for piezoelectric transducers, which are cheap and robust, but have a narrow bandwidth.

A problem that occurs in, for instance, indoor situations is that the direct response to a transmitted waveform is interfered by unwanted echoes. Reflective objects in the neighborhood of the object of interest cause multiple indirect paths between transmitter-object-receiver. Figure 1a shows an example. Here, the observed waveform is the result of the direct response of the object of interest and a second response due to an indirect path between object and transducers. Conventional matched filtering (MF) is based on a model that does not include multiple responses. The interference of a second response, however, is deadly for the performance of MF, as shown in Figure $1 \mathrm{~b}$.

Methods to overcome the problem of interfering responses are, for instance, data fitting ([1], [2] and [3]). This paper introduces a new method that can be regarded as a gener- alized version of the MF. The generalization is obtained by using a simple probabilistic model of the occurrence of multiple responses. An earlier paper [4] is similar in spirit, but is based on a much more complicated model involving a generalized Poisson point process to model an avalanche effect of arriving echoes. That model contained some parameters whose connotations are not immediately clear. The model proposed here is much simpler, and with less parameters. The meaning of these parameters is immediately clear. An elaborated discussion of the method described in this paper can be found in [2] and [3].

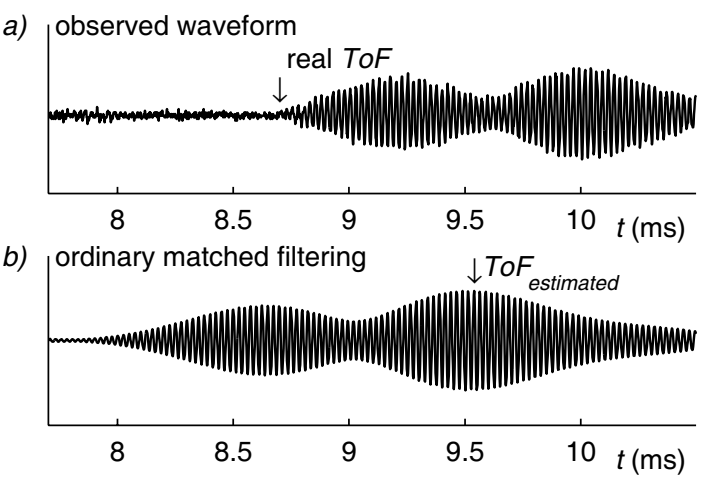

Figure 1. An observed waveform (a) and the corresponding result of the matched filter (b).

The paper starts with a short review on the conventional MF (Section II). The generalized version will be developed in Section III. Experiments are reported in Section IV. The paper finalizes with the conclusion.

\section{CONVENTIONAL MATCHED FILTERING}

Matched filtering assumes that the observed waveform consists of a delayed version of the nominal response $h(t)$ immersed in noise. Let $\tau$ be the ToF, and $a$ the attenuation, then $w(t)=a h(t-\tau)+n(t)$. Suppose that $z_{k}=w(k \Delta)$ is a time-discrete representation of the waveform. $\Delta$ is the sampling period. The observed waveform is represented by a vector $\mathbf{z}$ with elements $z_{k}$. Upon introduction of a vector $\mathbf{h}(\tau)$ with elements $h(k \Delta-\tau)$, the model becomes:

$$
\mathbf{z}=a \mathbf{h}(\tau)+\mathbf{n}
$$

Since the measurement noise is assumed to be Gaussian and white, with zero mean and standard deviation $\sigma_{n}$, the likelihood function of $\mathbf{z}$ is ( $c$ is a normalizing constant):

$$
p(\mathbf{z} \mid \tau)=\frac{1}{c} \exp \left(-\frac{1}{2 \sigma_{n}^{2}}(\mathbf{z}-a \mathbf{h}(\tau))^{T}(\mathbf{z}-a \mathbf{h}(\tau))\right)
$$


The MF seeks the $\tau$ that maximizes the likelihood (conform maximum likelihood estimation). The solution is:

$$
\hat{\tau}=\underset{\tau}{\arg \max } p(\mathbf{z} \mid \tau)=\underset{\tau}{\arg \max } \mathbf{z}^{T} \mathbf{h}(\tau)
$$

which can be written in the equivalent form:

$$
\hat{\tau}=\Delta \underset{k}{\arg \max }(w(k \Delta) * h(k \Delta))
$$

where $*$ denotes correlation. Thus, $\tau$ is found as the moment of maximum correlation between $w(t)$ and $h(t)$.

\section{EXTENDED MATCHED FILTERING}

The conventional matched filter cannot cope with the interference caused by secondary responses. The method that will be developed next is based on two assumptions:

- The direct path between the object of interest and the transducers is always shorter than the indirect paths of the secondary responses. This assumption implies that the secondary responses always come after the direct response.

- The secondary responses are mutually independent with respect to time-shifts and intensities.

The conventional MF models the likelihood as a Gaussian. The informative part of likelihood is found in the expectation vector, whereas the noise is described by the covariance matrix (which, in case of white noise, is proportional to the unity matrix). In the situation of having secondary responses we must find a way to embed these responses in the likelihood function. Our strategy will be to put all information in the covariance matrix (which now must depend on $\tau$ ). The expectation vector will be of no use then. In fact, it will be regarded as zero. Thus, in the new situation, the likelihood will be:

$$
p(\mathbf{z} \mid \tau)=\frac{1}{\sqrt{(2 \pi)^{K}|\mathbf{C}(\tau)|}} \exp \left(-\frac{1}{2} \mathbf{z}^{T} \mathbf{C}(\tau)^{-1} \mathbf{z}\right)
$$

$K$ is the number of samples in $\mathbf{z} . \mathbf{C}(\tau)$ is the covariance matrix.

In order to get computational feasibility, principal component analysis will be applied to reduce the number of components that make up the argument of the exponential in eq. (5). This will lead to a computational structure that can be regarded as a generalization of eq. (4).

\section{The covariance model}

In order to describe the waveform in terms of a (nonstationary) autocovariance function we model it as follows:

$$
\begin{aligned}
w(t) & =a(h(t-\tau)+r(t-\tau))+n(t) \\
r(t) & =\sum_{m} d_{m} h\left(t-\tau_{m}\right)
\end{aligned}
$$

$r(t)$ represents the secondary echoes. $\tau_{m}$ are random points in time. We limit the occurrence of an echo to an interval $0<\tau_{m}<T$, and assume a uniform distribution.
The attenuation factors $d_{m}$ are modeled as Gaussian random variables with zero mean and variance $\sigma_{d}^{2}$. If there are $M$ echoes, then the autocovariance function of $r(t)$ equals:

$$
C_{r}\left(t_{1}, t_{2}\right)=\frac{M \sigma_{d}^{2}}{T} \int_{\alpha=0}^{T} h\left(t_{1}-\alpha\right) h\left(t_{2}-\alpha\right) d \alpha
$$

The sampled version of $r(t-\tau)$, that is $r(k \Delta-\tau)$, can be brought in a vector $\mathbf{r}(\tau)$. The vector is zero mean and with a covariance matrix $\mathbf{C}_{\mathbf{r} \mid \tau}$ whose elements are:

$$
C_{\mathbf{r} \mid \tau}(n, m)=C_{r}(n \Delta-\tau, m \Delta-\tau)
$$

The term $a h(t-\tau)$ in (6) represents the direct response. The factor $a$ is an unknown parameter. Ideally, it is modeled by a probability density $p(a)$ which reflects our state of knowledge (and uncertainty) that we have about $a$. Unfortunately, it is difficult to assess this state of knowledge. We will take our ease, and assume that $a$ has a zero mean, Gaussian density with variance $\sigma_{a}^{2}$. Such an assumption is counterintuitive because negative and zero values of $a$ are not likely to occur. The only reason for modeling it in this way is that that enables a tractable solution. We simply hope that the actual density of $a$ does not influence the solution too much. Note that in the conventional matched filter, the actual value of $a$ didn't have any influence either.

With the zero mean, Gaussian assumption of $a, a h(t-\tau)$ also becomes zero mean, and its autocovariance function becomes $\sigma_{a}^{2} h\left(t_{1}-\tau\right) h\left(t_{2}-\tau\right)$. The time-discrete version $a \mathbf{h}(\tau)$ is associated with a covariance matrix $\mathbf{C}_{\mathbf{h} \mid \tau}=\sigma_{a}^{2} \mathbf{h}(\tau) \mathbf{h}^{T}(\tau)$.

The sampled version $\mathbf{z}$ of the observed waveform consists of the direct response, the secondary responses and the noise. Taken together, the covariance matrix of $\mathbf{z}$ becomes:

$$
\mathbf{C}(\tau)=\sigma_{a}^{2}\left(\mathbf{h}(\tau) \mathbf{h}^{T}(\tau)+\mathbf{C}_{\mathbf{r} \mid \tau}\right)+\sigma_{n}^{2} \mathbf{I}
$$

Strictly speaking, $\mathbf{z}$ is not Gaussian. However, if we assume that a sufficient number of secondary responses pile up, then the central limit theorem applies, and $\mathbf{z}$ is approximately Gaussian. In that case, the optimal estimate of $\tau$ is the one that maximizes eq. (5). The procedure can be simplified to maximizing:

$$
\Lambda(\mathbf{z} \mid \tau) \stackrel{\text { def }}{=}-\mathbf{z}^{T} \mathbf{C}(\tau)^{-1} \mathbf{z}
$$

because the determinant $|\mathbf{C}(\tau)|$ does not depend on $\tau$.

\section{Principal Component Analysis}

Since the registration interval of $w(t)$ is so large that it may easily comprise 10000 samples or more, the direct maximization of $\Lambda(\mathbf{z} \mid \tau)$ is not feasible. We apply principal component analysis to extract a linear subspace of $\mathbf{z}$. The subspace must be selected such that it retains most of the information on $\tau$. For that purpose, we decompose $\mathbf{C}(\tau)$ in eigenvalues $\lambda_{k}(\tau)$ and eigenvectors $\mathbf{u}_{k}(\tau)$ : 


$$
\mathbf{C}(\tau)=\sum_{k=1}^{K} \lambda_{k}(\tau) \mathbf{u}_{k}(\tau) \mathbf{u}_{k}(\tau)^{T}
$$

so that eq. (10) can be molded in the equivalent form:

$$
\Lambda(\mathbf{z} \mid \tau)=-\sum_{k=1}^{K} \frac{\left(\mathbf{z u}_{k}(\tau)\right)^{2}}{\lambda_{k}(\tau)}
$$

The computational savings are obtained by discarding all terms in eq. (12) that do not depend much on $\tau$.

The selection of components can be done by realizing that $\sum_{k=1}^{K}\left(\mathbf{z}^{T} \mathbf{u}_{n}(\tau)\right)^{2}=\|\mathbf{z}\|^{2}$, so that:

$$
\Lambda(\mathbf{z} \mid \tau)=\sum_{k=1}^{K} \frac{\lambda_{k}(\tau)-\sigma_{n}^{2}}{\lambda_{k}(\tau) \sigma_{n}^{2}}\left(\mathbf{z}^{T} \mathbf{u}_{k}(\tau)\right)^{2}-\frac{\|\mathbf{z}\|^{2}}{\sigma_{n}^{2}}
$$

The term containing $\|\mathbf{z}\|$ does not depend on $\tau$ and can be omitted. Eq. (13) shows that the components $\mathbf{z}^{T} \mathbf{u}_{k}(\tau)$ for which the factors $\gamma_{k}=\left(\lambda_{k}(\tau)-\sigma_{n}^{2}\right) /\left(\lambda_{k}(\tau) \sigma_{n}^{2}\right)$ are small can be discarded without much loss of accuracy.
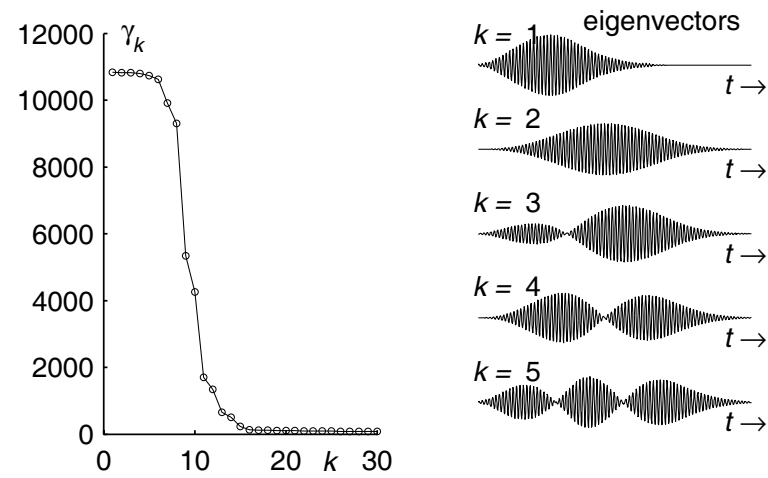

Figure 2. The factors $\gamma_{k}$ and the first five corresponding eigenvectors.

Figure 2 is an example showing that the sequence $\gamma_{k}$ indeed rapidly decreases. About 8 components suffice to calculate $\Lambda(\mathbf{z} \mid \tau)$. The figure also shows the eigenvectors $\mathbf{u}_{k}(0)$ corresponding to the five most important eigenvalues. The first eigenvector has a strong resemblance with the direct response.

\section{The Computational Structure}

A fast implementation of the procedure discussed in the previous section is obtained by the observation that:

- The eigenvalues $\lambda_{k}(\tau)$ do not depend on $\tau$.

- A change of $\tau$ causes only a shift of elements in the eigenvectors $\mathbf{u}_{k}(\tau)$. Suppose that $u_{k, m}(\tau)$ is the $m$-th element from $\mathbf{u}_{k}(\tau)$. Then: $u_{k, m}(\tau+\Delta)=u_{k, m-1}(\tau)$.

These properties result from the fact that a change of $\tau$ only causes a shift of the elements in the covariance matrix $\mathbf{C}(\tau)$ along the rows and the columns.

The properties prevent the need for calculating the eigenvectors and eigenvalues of $\mathbf{C}(\tau)$ for every $\tau$. It suffices to calculate the eigenvectors and eigenvalues for $\mathbf{C}(0)$. De- fining $\lambda_{k}=\lambda_{k}(0)$ and $\mathbf{u}_{k}=\mathbf{u}_{k}(0)$, and denoting the elements of $\mathbf{u}_{k}$ by $u_{k, m}$ the computational structure of eq. (13) is equivalent to:

$$
\Lambda(\mathbf{z} \mid m \Delta)=\sum_{k=1}^{J} \gamma_{k}\left(z_{m} * u_{k, m}\right)^{2}
$$

Here, we have assumed that the eigenvalues/eigenvectors are ordered according to their importance indicated by $\gamma_{k}$ (as in Figure 2), and that the first $J$ components are selected.

Note that in this context, the eigenvectors, as shown in Figure 2, can be interpreted as the impulse responses of a filter bank. The output signals of the bank are squared, multiplied by a weighting factor $\gamma_{k}$, and finally summed together yielding $\Lambda(\mathbf{z} \mid m \Delta)$.

If only one component is selected, $J=1$, the method coincides with the conventional matched filter. This is so, because the most important eigenvector of $\mathbf{C}(\tau)$ is proportional to $\mathbf{h}(\tau)$. The impulse responses of the other filters match the echoes.

As an example, consider the observed waveform shown in Figure 1. The conventional matched filter does not work properly here, and its error is about $0.8 \mathrm{~ms}$. The same waveform has been processed according to the newly proposed method. Results are shown in Figure 3. It can be seen that the response of the first filter resembles the output of the matched filter shown in Figure 1. Without further processing the error would be again about $0.8 \mathrm{~ms}$. However, the contributions of the three other filters compensates for the shift caused by the secondary responses. The remaining error is less than $0.1 \mathrm{~ms}$.
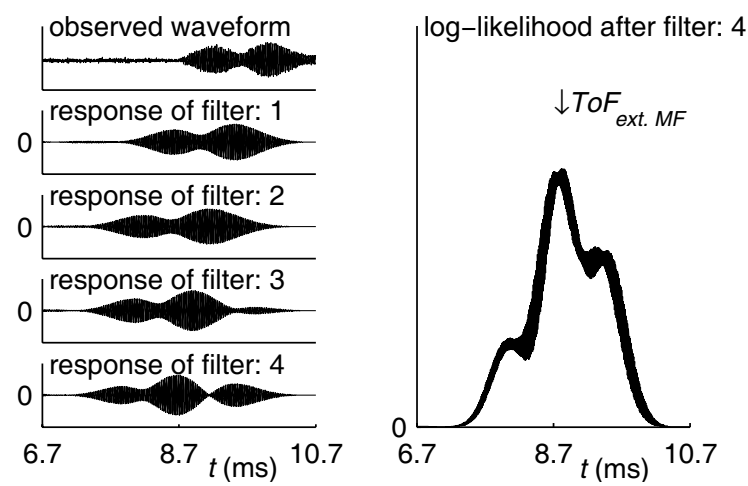

Figure 3. (left): the responses of the first four filters applied to the observed waveform shown on top. (right): the resulting log-likelihood $\Lambda(\mathbf{z} \mid \tau)$.

\section{EXPERIMENTS}

The purpose of the chapter is to demonstrate the qualities of the extended matched filter. We do this by comparing its performance with the one of the ordinary matched filter. The set-up that was used for the experimentation consisted of a piezoelectric transmitter that is placed in front of a piezoelectric receiver. Using this set-up, a dataset con- 
sisting of 150 records of waveforms was acquired in indoor situations, i.e. in different rooms, at different locations, for different heights above the floor, and for different distances between transmitter and receiver. The transmitted tone burst consisted of 20 periods of a sine wave of 40 $\mathrm{kHz}$. The sampling period was $\Delta=2 \mu \mathrm{s}$. Each recorded waveform is accompanied by a reference ToF indicating the true value of the ToF. The uncertainty of the reference ToF is estimated at $10 \mu \mathrm{s}$. In addition to the 150 records there is also a registration of the nominal response $h(t)$ obtained in an anechoic room.

\section{Evaluation}

The quality of an estimator is assessed by means of its bias and its variance. The overall bias is a constant error independent from the real ToF. If known, it can be compensated for. We used 3-fold cross validation (see below) to assess the overall bias and the variance. It appeared that for both the ordinary MF and the extended MF the bias was small compared with the random error. Therefore, we used the sample variance as a performance criterion during the optimization.

The design parameters of the extended matched filter are:

- The signal-to-noise ratio defined as $S N R=\sigma_{a}^{2} / \sigma_{n}^{2}$.

- The length $T$ of the interval where echoes can appear.

- The relative echo strength, i.e. $M \sigma_{d}^{2}$.

- The number $J$ of selected components.

- The window size, i.e. the dimension of the covariance matrices (and thus the length of the eigenvectors $\mathbf{u}_{k}$ ).

These parameters must be selected such that the operator has maximal performance, i.e. minimal sample variance.

\section{3-fold cross validation}

A straightforward minimization of the sample variance by tuning the design parameters in a scheme as indicated in Figure 4 is biased since the same dataset is used for both training (optimization) and evaluation. It would be better to split the dataset into two non-overlapping partitions, one for training, and one for evaluation. Such a partitioning does not use the dataset efficiently; a major drawback if the dataset is small.

Instead, we used 3-fold cross validation to optimize the design parameters and to evaluate the results. The proce-

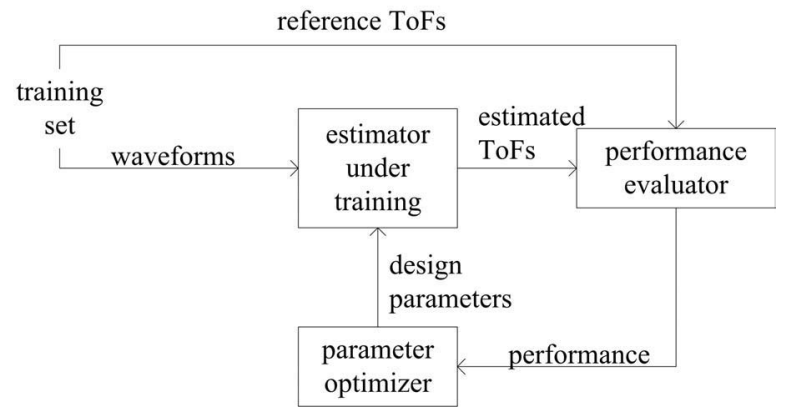

Figure 4. Optimization and evaluation of an estimator using a training set (from [2]). dure is to randomly split the dataset into 3 subsets $T_{1}, T_{2}$ and $T_{3}$. First, $T_{1}$ and $T_{2}$ are pooled and used for training while $T_{3}$ is used for evaluation. Next, $T_{2} \cap T_{3}$ is used for training and $T_{1}$ for evaluation. After that, $T_{3} \cap T_{1}$ is used for training and $T_{2}$ for evaluation. The final performance is obtained by averaging the three sample variances.

\section{Results}

The results are shown in Table 1. The variance is the average of the three sample variances (see above), corrected with $100 \mu s^{2}$ to account for the uncertainty in the reference ToFs. The term 'bias' refers here to the residual uncertainty of the constant error, after application of bias compensation.

Table 1. Estimated performance

\begin{tabular}{|c|c|c|c|}
\hline & & conventional MF & extended MF \\
\hline variance & $\mu s^{2}$ & 14279 & 491 \\
bias & $\mu s$ & 10 & 2 \\
\cline { 1 - 2 } RMS & $\mu s$ & $119 \pm 7$ & $22 \pm 1.4$ \\
\hline
\end{tabular}

\section{CONCLUSION}

Extended matched filtering, as introduced in this paper, models the signal in terms of a non-stationary autocovariance function. This is in contrast with the ordinary matched filter which models the signal in terms of an expectation. Working out the autocovariance model leads to a ToF estimator that uses a bank of filters rather than just one.

Using a dataset of 150 records of waveforms acquired under various circumstances it was shown that the new method outperforms the ordinary matched filter.

\section{REFERENCES}

[1] X. Li, R. Wu, S. Rasmi, J. Li, L.N. Cattafesta, M. Sheplak, "Acoustic Proximity Ranging in the Presence of Secondary Echoes", IEEE Tr. Instrumentation and Measurement, Vol. 52, No. 5, pp. 1593-1605, October 2003.

[2] F. van der Heijden, R.P.W. Duin, D. de Ridder, D.M.J. Tax, "Classification, Parameter Estimation and State Estimation - An Engineering Approach using Matlab", Wiley, pp. 319-339, Chichester (2004).

[3] S. van Koningsveld, "Time-of-Flight estimation in reflective environments", MSc thesis, University of Twente, Laboratory for Measurement and Instrumentation, The Netherlands, (2003).

[4] F. van der Heijden, G. Túquerres, P.P.L. Regtien, "Time-of-flight estimation based on covariance models", Measurement Science and Technology, vol. 14, no. 8, pp.1295-1304, August 2003. 